\title{
ON THE NEGATIVE SOLUTIONS OF $L_{p}$-BUSEMANN-PETTY PROBLEM
}

\author{
WEIDONG WANG, HAi Li AND ZHONGHUAN SHEN
}

\begin{abstract}
Intersection bodies led to the solutions of Busemann-Petty problem by Lutwak. Associated with Haberl and Ludwig's $L_{p}$-intersection bodies, Yuan and Cheung researched related the $L_{p}$-Busemann-Petty problem. In this paper, we sequentially study $L_{p}$-Busemann-Petty problem of $L_{p}$-intersection bodies and give its two negative forms.
\end{abstract}

Mathematics subject classification (2010): 52A20, 52A39, 52A40.

Keywords and phrases: $L_{p}$-intersection body, $L_{p}$-Busemann-Petty problem, negative solution.

\section{REFERENCES}

[1] R. J. Gardner, Intersection bodies and the Busemann-Petty problem, Trans. Amer. Math. Soc., 342 (1994), 1: 435-445.

[2] R. J. Gardner, A positive answer to the Busemann-Petty problem in three dimensions, Ann. of Math., 140 (1994), 2: 435-447.

[3] R. J. Gardner, Geometric Tomography, Second ed., Cambridge Univ. Press, Cambridge, 2006.

[4] R. J. Gardner, A. Koldlbsky and T. Schlumprecht, An analytic solution to the Busemann-Petty problem on sections of convex bodies, Ann. of Math., 149 (1999), 691-703.

[5] C. Haberl, $L_{p}$ intersection bodies. Adv. Math., 217 (2008), 6: 2599-2624.

[6] C. Haberl and M. Ludwig, A characterization of $L_{p}$ intersection bodies, Int. Math. Res. Not., 2006 (2006), Art ID 10548, 29 pages.

[7] A. Koldlbsky, Intersection bodies and the Busemann-Petty problem, C. R. Acad. Sci. Paris Ser. I Math., 325 (1997), 11: 1181-1186.

[8] A. Koldlbsky, Intersection bodies in $\mathbb{R}^{4}$, Adv. Math., 136 (1998), 1: 1-14.

[9] A. Koldlbsky, Intersection bodies, positive definite distributions, and the Busemann-Petty problem, Amer. J. Math., 120 (1998), 4: 827-840.

[10] E. Lutwak, Intersection bodies and dual mixed volumes, Adv. Math., 71 (1988), 531-538.

[11] Y. N. Pei and W. D. Wang, A type of Busemann-Petty problems for general $L_{p}$-intersection bodies, Wuhan University Journal of Natural Sciences, 20 (2015), 6: 471-475.

[12] B. Rubin and G. Y. Zhang, Generalizations of the Busemann-Petty problem for sections of convex bodies, J. Funct. Anal., 213 (2004), 473-501.

[13] R. Schneider, Convex Bodies: The Brunn-Minkowski theory, 2nd edn, Cambridge University Press, Cambridge, 2014.

[14] F. E. Schuster, Valuations and Busemann-Petty type problems, Adv. Math., 219 (2008), 344-368.

[15] Z. H. Shen, Y. N. Li and W. D. Wang, $L_{p}$-dual geominimal surface areas for the general $L_{p}$ intersection bodies, J. Nonlinear Sci. Appl., 10 (2017), 7: 3519C3529.

[16] J. Y. Wang and W. D. Wang, General $L_{p}$-dual Blaschke bodies and the applications, J. Inequal. Appl., (2015) 2015: 233: 11 pages.

[17] J. Y. Wang and W. D. Wang, $L_{p}$-dual affine surface area forms of Busemann-Petty type problems, Proc. Indian Acad. Sci. (Math. Sci.), 125 (2015), 1: 71-77.

[18] W. D. Wang and Y. N. Li, Busemann-Petty problems for general $L_{p}$-intersection bodies, Acta Math. Sin. (English Series), 31 (2015), 5: 777-786.

[19] W. Y. Yu, D. H. Wu and G. S. Leng, Quasi L L -intersection bodies, Acta Math. Sinica, 23 (2007), 11: 1937-1948. 
[20] J. Yuan and Wingsum Cheung, $L_{p}$-intersection bodies, J. Math. Anal. Appl., 339 (2008), 2: 14311439.

[21] G. Y. Zhang, Intersection bodies and the four-dimensional Busemann-Petty problem, Duke Math. J., 71 (1993), 233-240.

[22] G. Y. Zhang, Centered bodies and dual mixed volumes, Trans. Amer. Math. Soc, 345 (1994), 2: $777-$ 801.

[23] G. Y. Zhang, Intersection bodies and the Busemann-Petty inequalities in $\mathbb{R}^{4}$, Ann. of Math., 140 (1994), 2: 331-346.

[24] G. Y. Zhang, A positive solution to the Busemann-Petty problem in $\mathbb{R}^{4}$, Ann. of Math., 149 (1999), 2: 535-543. 